Esta revista forma parte del acervo de la Biblioteca Jurídica Virtual del Instituto de Investigaciones Jurídicas de la UNAM

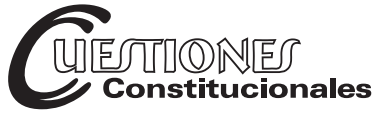

Revista Mexicana de Derecho Constitucional Núm. 44, Enero-Junio 2021

ISSN (versión electrónica): 2448-4881

\title{
Internet, redes sociales y libertad de expresión
}

\author{
Internet, social media and freedom of expression
}

Recepción: 9 de septiembre de 2019

Aceptación: 31 de enero 2020

\author{
Mauricio Augusto CALCANEO MONTS*
}

RESUMEN: El texto expone y explica las implicaciones que para la libertad de expresión tiene el surgimiento de la regulación proveniente de las empresas de redes sociales. Lo que adquiere relevancia ante la implosión del Internet y su concentración en algunas redes sociales, que en esa medida controlan la libertad de expresión en línea de sus millones de usuarios alrededor del mundo. La regulación de la libertad de expresión en el siglo XXI es un triángulo, donde los sujetos están sometidos a la normatividad estatal propia del siglo XX y a las reglas de las empresas de redes sociales. Es un cambio en los cimientos de la libertad de expresión, que requiere de una amplia reflexión sobre su significado, alcances y los ajustes jurídicos requeridos, donde cobra importancia la propuesta de aplicar el derecho internacional de los derechos humanos a la regulación empresarial de la libertad de expresión en Internet.
ABSTRACT: The text sets out and explain the implications for freedom of expression on the emergence of regulation from social media companies. What becomes relevant in the face of the implosion of Internet and its concentration on some social networks, which to that extent control the online freedom of expression of its millions of users around the world. The regulation of freedom of expression in the $21^{\text {st }}$ century is a triangle, where the subjects are submitted to the state regulations and the rules of social media companies. It is a change in the foundations of freedom of expression that requires broad reflection on its meaning, scopes and required legal adjustments, where the proposal to apply international human rights law to the business regulation of freedom of expression on the Internet becomes important.

* Candidato a doctor por el Posgrado en Ciencias Políticas y Sociales de la UNAM, México. Correo electrónico: calcaneomonts@yahoo.com ORCID: 0000-0001-96047695 . 
Esta revista forma parte del acervo de la Biblioteca Jurídica Virtual del Instituto de Investigaciones Jurídicas de la UNAM

Palabras clave: Internet, libertad de expresión, redes sociales, regulación, derechos humanos.
Keywords: Internet, Free Speech, Social Media, Regulation, Human Rights.

\begin{abstract}
SUMARIO: I. Introducción. II. La implosión del Internet y el control de las redes sociales. III. Los guardianes de la libertad de expresión en Internet. IV. El derecho a la libertad de expresión en el siglo XX y el siglo XXI, el tránsito del modelo dualista al pluralista. V. La libertad de expresión en Internet y el derecho internacional de los derechos humanos. VI. Conclusiones. VII. Fuentes consultadas.
\end{abstract}

\title{
I. INTRODUCCIÓN
}

Han transcurrido cinco lustros desde que inició el uso masivo del Internet, lapso en el cual el número de usuarios pasó de representar menos del uno por ciento de la población mundial (16 millones de personas o $0.4 \%$ de dicha población en 1995), a casi dos tercios de quienes habitan el planeta tierra en 2020 ( $62 \%$ de éstos o 4,833 millones de individuos). ${ }^{1}$

El Internet conecta a millones de seres humanos prácticamente a todo lo largo y ancho del orbe, eliminando así las barreras espaciales y temporales existentes con antelación. Se trata, sin duda alguna, de un cambio de gran envergadura en el ámbito tecnológico y de las comunicaciones, con hondas repercusiones en la forma en que los seres humanos se expresan e interactúan.

El Internet, o más precisamente las redes sociales, se han erigido en el espacio por antonomasia donde las personas publican, comentan, debaten y comparten ideas, informaciones, videos, fotografías y demás. El Internet y las redes sociales son, como dijo Anthony M. Kennedy ex juez de la Corte Suprema de Estados Unidos, la "plaza pública moderna" (citado por Citrón y Richards: 1355) o la nueva plaza pública.

El Internet despertó en un inicio amplias esperanzas por las posibilidades que brindaba para ensanchar la libertad de expresión, la participación política y la democracia alrededor del mundo. A grado tal era la fe que

\footnotetext{
1 Disponible en: https://www.internetworldstats.com/emarketing.htm (fecha de consulta: 2 de septiembre de 2020).

Cuestiones Constitucionales, Núm. 44, Enero-Junio 2021

ISSN: $2448-4881$
} 
Esta revista forma parte del acervo de la Biblioteca Jurídica Virtual del Instituto de Investigaciones Jurídicas de la UNAM

se le consideró, junto con las computadoras, los teléfonos celulares y las redes sociales, como parte de las denominadas tecnologías de la liberación (Diamond, 2010).

La situación sufrió un vuelco y el optimismo ha dado paso a la preocupación (Diamond, 2019), principalmente por una serie de aspectos tales como: la extracción de ingentes volúmenes de información de las personas y su uso con fines predictivos y persuasivos en los más distintos ámbitos de la vida social (Calcaneo, 2019); la utilización bastante extendida de técnicas de manipulación y desinformación en línea principalmente por gobiernos de corte autoritario, aunque también por gobiernos democráticos y por particulares (Freedom House, 2018); y, más recientemente, el creciente poder de las empresas de redes sociales con respecto a un conjunto de temas entre los que destaca la libertad de expresión en línea.

En efecto, cada vez son más abiertas las decisiones que las empresas de redes sociales están tomando, cotidiana y unilateralmente, con relación a la libertad de expresión de sus usuarios. Se trata de un conjunto de medidas que, en los hechos, norman lo que se dice en el Internet, lo que incluye acciones como eliminar y bloquear cuentas, restringir mensajes, videos, entre otras. Es decir, están regulando, sin rendir cuentas a nadie y en la más completa opacidad, la libertad de expresión de los millones de personas que hacen uso de sus servicios.

El presente texto tiene como propósito exponer y explicar las implicaciones que para la libertad de expresión tiene el surgimiento de un nuevo tipo de regulación de por parte de las empresas de redes sociales, en una realidad en la que el Internet y las redes sociales se han erigido en la nueva plaza pública. Al tiempo de dar cuenta de las propuestas desarrolladas desde el derecho internacional de los derechos humanos para limitar el gran poder que en la materia tienen un puñado de empresas de redes sociales.

El documento se divide en cuatro apartados correspondientes a: en primer lugar, la exposición sobre la implosión en el uso del Internet y el control de éste por las empresas de redes sociales, lo que da a éstas un enorme poder sobre lo que se puede decir o no en línea; en segundo lugar, se explican los sistemas de revisión de contenidos que las empresas de redes sociales han desarrollado en su interior para regular la libertad de expresión de sus millones de usuarios, lo que ha convertido a dichas empresas en los nuevos guardianes de la libertad de expresión; en tercer lugar, se explican las diferencias, implicaciones y retos que significan los dos tipos 
Esta revista forma parte del acervo de la Biblioteca Jurídica Virtual del Instituto de Investigaciones Jurídicas de la UNAM

de regulaciones paralelas existentes en la actualidad en materia de libertad de expresión, la dualista del siglo XX y la empresarial del siglo XXI; y, en cuarto lugar, se revisa el planteamiento que pugna por aplicar el derecho internacional de los derechos humanos en materia de libertad de expresión a la regulación de contenidos por parte de las empresas de redes sociales, para así circunscribir el creciente y discrecional poder de éstas en relación con la libertad de expresión de su ingente número de usuarios.

\section{LA IMPLOSIÓN DEL INTERNET Y EL CONTROL DE LAS REDES SOCIALES}

En 1995, 16 millones de personas alrededor del orbe usaban el Internet, para 2020 dicha cifra ascendió a aproximadamente 4 mil 800 millones de personas, es decir, un poco más de seis de cada diez habitantes del planeta tierra usaban el Internet. Es un crecimiento exponencial en un cuarto de siglo, que se acompaña de una clara y sostenida tendencia al alza. A lo que se suma el incremento sostenido en el tiempo que las personas destinan diariamente al uso del Internet, en 2011 eran 75 minutos, para 2020 son 181 minutos y se calculan 192 minutos para $2021 .^{2}$ En una década se pasó de un consumo diario de Internet por persona de 1 hora con 15 minutos a tres horas con un minuto, un aumento de una hora con cuarenta y seis minutos por individuo. Es un doble proceso en el que cada vez son más los sujetos que acceden al Internet, a la vez que los mismos pasan mayor tiempo navegando en la red.

La implosión en la cantidad de usuarios y el mayor tiempo invertido en el Internet ha ido acompañada por su concentración en unas cuantas empresas de redes sociales. En 2020, 3 mil 600 millones de personas alrededor del mundo eran usuarias de redes sociales, el equivalente a la mitad de la población mundial. Facebook por sí solo reportó 2 mil 700 millones de usuarios, ${ }^{3}$ YouTube 1 mil 860 millones $^{4}$ y Twitter 330 millones. ${ }^{5}$ Más

2 Disponible en: https://www.statista.com/statistics/319732/daily-time-spent-online-device/ (fecha de consulta: 3 de septiembre de 2020).

3 Disponible en: https://www.statista.com/statistics/264810/number-of-monthlyactive-facebook-users-worldwide/ (fecha de consulta: 4 de septiembre de 2020).

4 Disponible en: https://www.statista.com/topics/2019/youtube/ (fecha de consulta: 2 de agosto de 2019).

5 Disponible en: https://www.statista.com/statistics/282087/number-of-monthlyactive-twitter-users/(fecha de consulta: 4 de septiembre de 2020).

Cuestiones Constitucionales, Núm. 44, Enero-Junio 2021

ISSN: $2448-4881$ 
Esta revista forma parte del acervo de la Biblioteca Jurídica Virtual del Instituto de Investigaciones Jurídicas de la UNAM

todavía, en 2019 el tiempo promedio diario que las personas destinaban al uso de las redes sociales ascendía a 144 minutos o 2 horas con 24 minutos. La situación es diáfana: las redes sociales concentran el grueso de quienes acceden al Internet y del tiempo que estos destinan a explorarlo.

El Internet, o más precisamente las redes sociales, se han convertido en la nueva plaza pública, en el espacio por excelencia para el intercambio de ideas, para publicar y compartir opiniones, pensamientos, fotos, videos y demás. Sin embargo, esto ha ido acompañado de un proceso de control del Internet por un puñado de empresas de redes sociales, lo que se explica por la centralización y el desvanecimiento de las diferentes capas de la arquitectura del Internet como advierte Jonathan Zittrain (2018):

Primero, el tema de la centralización... navegar por Internet puede ahora significar simplemente saltar entre los servidores de alojamiento de los servicios de Amazon... Segundo, las capas anteriormente separadas de la arquitectura del Internet se están difuminando... el éxito de algunas empresas ha creado nuevas plataformas exclusivas integradas. Mucha gente no está usando en realidad el Internet, sino más bien revolotean entre un pequeño puñado de aplicaciones totalizadoras como Facebook y Google (Facebook y Google: 871).

Unas cuantas empresas de redes sociales se han hecho del control del Internet y, por ende, de lo que se dice, se comparte y se publica, de la libertad de expresión en la nueva plaza pública. Olvidada quedó la promesa del Internet libre, sin intermediarios, donde los individuos podían generar sus propios contenidos y compartirlos sin limitación alguna.

En la sociedad algorítmica, como Jack M. Balkin (2018b) denomina a la presente etapa donde las plataformas de redes sociales gobiernan a las personas, se ponen a prueba:

Las condiciones prácticas del discurso, así como las entidades que lo controlan, limitan y censuran... Primero, el discurso digital fluye a través de una elaborada infraestructura privada de comunicación. Hoy, nuestra capacidad práctica para expresarnos está sujeta a las decisiones de los propietarios de infraestructura privada, que gobiernan los espacios digitales en los que las personas se comunican entre sí. Este es el problema de la gobernanza privada del discurso (Balkin, 2018b: 1153). 
Esta revista forma parte del acervo de la Biblioteca Jurídica Virtual del Instituto de Investigaciones Jurídicas de la UNAM

Las empresas de redes sociales al detentar la propiedad de la infraestructura de comunicación a través de la cual las personas llevan a cabo sus interacciones en el mundo digital, se han convertido, como se muestra en el siguiente apartado, en los guardianes de la libertad de expresión en línea.

\section{LOS GUARDIANES DE LA LIBERTAD DE EXPRESIÓN EN INTERNET}

Mark Zuckerberg (2019), fundador y director ejecutivo de Facebook, expresó abiertamente el enorme poder que su empresa de redes sociales ejerce sobre la libre expresión en línea y los pasos a seguir a tal respecto:

Los legisladores a menudo me dicen que tenemos demasiado poder sobre el discurso, y francamente estoy de acuerdo. He llegado a creer que no debemos tomar tantas decisiones importantes sobre lo que se dice. Por lo tanto, estamos creando un organismo independiente para que las personas puedan apelar nuestras decisiones. También estamos trabajando con los gobiernos... para garantizar la efectividad de los sistemas de revisión de contenido.

En efecto, Facebook cuenta, a decir del propio Zuckerberg (2018), con un sistema de gobernanza del contenido que circula por dicha red social, que revisa cada día dos millones de piezas de contenido y que se integra por: los estándares de la comunidad o las reglas que determinan qué contenido se mantiene y cuál es removido; las pautas internas que reducen el nivel de subjetividad de las decisiones que se adoptan y generan consistencia en las mismas; un equipo global de cerca de 30 mil individuos encargados de moderar el contenido, con 10 oficinas alrededor del mundo y que también implica una serie de retroalimentaciones con académicos y especialistas en materia de libertad de expresión y seguridad; y el uso de la inteligencia artificial para detectar y remover de manera automática y preventiva el contenido que se considera problemático.

A lo que se sumó, más recientemente, la creación y puesta en funcionamiento de lo que se ha dado por denominar como Consejo Asesor de Contenido de Facebook, que es una instancia independiente conformada por especialistas y que está encargada de analizar los casos más sobresalientes y controvertidos en materia de libertad de expresión que deriven del sistema de revisión interno de la empresa, pudiendo revertir las deci- 
Esta revista forma parte del acervo de la Biblioteca Jurídica Virtual del Instituto de Investigaciones Jurídicas de la UNAM

siones tomadas por ésta sobre los contenidos que se permiten circular y los que son retirados. Lo que se dice dotará de legitimidad, transparencia y equidad al sistema de revisión de contenidos Facebook (Zuckerberg, 2018; Facebook, 2019; y Clegg, 2019).

Se trata, en síntesis, de un sistema de revisión del contenido que los usuarios pretenden publicar en Facebook, que posibilita a dicha empresa a determinar por medio de seres humanos y de inteligencia artificial, ya sea de manera reactiva y proactiva, qué se queda y qué se va de la plataforma digital. Es decir, Facebook está tomando todos los días y en todo momento decisiones con respecto a lo que sus usuarios pueden o no expresar, sobre la libertad de expresión de estos. Son dos millones de piezas de publicaciones que día a día pasan por el tamiz del sistema de revisión de contenidos de Facebook, quien decide unilateralmente, sin rendir cuentas a nadie y sin las garantías mínimas el alcance de la libertad de expresión de los millones de personas que hacen uso de sus servicios.

Sin embargo, este tipo de acciones de las plataformas en línea con relación a la libertad de expresión de sus usuarios no es, ni de lejos, exclusiva de Facebook. Las distintas empresas de redes sociales están adoptando, de diferentes maneras, un conjunto de medidas para, de facto, normar los contenidos que sus usuarios publican y comparten. Lo que incluye, por ejemplo, eliminar y bloquear cuentas, restringir la publicación de mensajes y videos, entre otras acciones. Como afirma Kate Klonick (2018: 1601) "aunque parece que cualquier usuario del Internet puede publicar libre e instantáneamente en línea, muchas plataformas de publicación de contenido moderan activamente lo publicado por sus usuarios".

Twitter y YouTube, al igual que Facebook, han desarrollado sus respectivos sistemas de regulación del contenido que, como su nombre lo indica, son entramados privados de moderación del discurso en línea que se sustentan en las leyes norteamericanas sobre libertad de expresión en Internet (primordialmente la sección 230 de la Ley de Decencia en las Telecomunicaciones), en la responsabilidad corporativa, pero sobre todo en la necesidad económica de generar un ambiente propicio para los usuarios pues de lo contrario éstos abandonarían dichas plataformas con efectos adversos en su rentabilidad económica. Sistemas de moderación que se asemejan a un sistema legal ya que cuentan con un conjunto normativo establecido con antelación; con procedimientos ex ante y ex post para la determinación de contenido contrario a las reglas, los valores democráticos y culturales; 
Esta revista forma parte del acervo de la Biblioteca Jurídica Virtual del Instituto de Investigaciones Jurídicas de la UNAM

con una serie de personas encargadas de aplicar esas reglas y que son entrenadas para el ejercicio de dicha función; y que se ven influenciados por factores internos y externos para su modificación o ajuste (Klonick, 2018).

Las redes sociales cuentan, desde ya, con toda una serie de reglas, procedimientos, tecnologías y personas encargadas de moderar, de manera previa o posterior y en la más absoluta opacidad y discrecionalidad, las publicaciones de sus usuarios. Las plataformas en línea son los nuevos gobernadores o guardianes de la libertad de expresión en línea (Klonick, 2018).

Los ejemplos abundan sobre las decisiones de las redes sociales en materia de libertad de expresión en el mundo digital, por citar solamente uno de los casos más emblemáticos está la decisión de Twitter de marcar con advertencias el contenido que se consideró "incorrecto" de varios mensajes del presidente de los Estados Unidos, Donald Trump (Coger y Alba, 2020), y después al prohibir el funcionamiento de la cuenta de la campaña presidencial de éste hasta que se retirara un video que consideraban contenía información "falsa" (Dodds, 2020). Twitter y las demás redes sociales fungen claramente como los nuevos guardianes de la libertad de expresión en el Internet.

El siglo XXI con el desarrollo del Internet y el control de éste por parte de las empresas de redes sociales coloca a la libertad de expresión en una nueva encrucijada, una encrucijada en la que unos particulares, las referidas empresas de redes sociales, que detentan un enorme poder derivado de su dominio sobre la infraestructura en línea, están decidiendo por sí mismas, en la más completa opacidad y sin rendir cuentas a nadie, qué es lo que puede o no circular en Internet, qué fluye y qué se excluye, esto es, sobre la libertad de expresión de los millones de usuarios de la nueva plaza pública.

En un mundo cada vez más volcado a lo digital, es un poder privado transnacional sin control ni restricción alguna, opaco y que no rinde cuentas, que tiene enormes implicaciones en uno de los pilares de los regímenes democráticos como lo es la libertad de expresión. Lo que pone en entredicho el papel de los Estados-nación y de la normatividad nacional e internacional desarrollada en el siglo XX en materia de libertad de expresión y opinión, y que abre paso a un nuevo arreglo en el que estas empresas de redes sociales adquieren gran relevancia en la materia. 
Esta revista forma parte del acervo de la Biblioteca Jurídica Virtual del Instituto de Investigaciones Jurídicas de la UNAM

\section{EL DERECHO A LA LIBERTAD DE EXPRESIÓN EN LOS SIGLOS XX Y XXI. EL TRÁNSITO DEL MODELO DUALISTA AL PLURALISTA}

El derecho a la libertad de expresión está ampliamente reconocido y regulado tanto en el ámbito internacional como el nacional por lo menos desde mediados del siglo XX. Es un derecho a la libertad de expresión que fue concebido principalmente como una protección, como un límite, de los individuos (llámense sujetos, medios de comunicación masiva y demás) frente al actuar arbitrario del Estado. De manera que la libertad de expresión de los individuos está tutelada por una serie de derechos en su favor, con las correspondientes obligaciones para los Estados.

La situación de la libertad de expresión en el siglo XXI sufre un cambio profundo con el vuelco hacia lo digital y la aparición de las empresas de redes sociales como un nuevo actor que se autorregula y a la vez regula a sus usuarios. De forma tal que los individuos están sujetos ahora a dos tipos distintos de regulaciones en materia de libertad de expresión, la propia del modelo dualista del siglo XX y la derivada de la regulación empresarial del siglo XXI.

Jack M. Balkin (2018a) ha hecho hincapié en el cambio regulatorio en materia de libertad de expresión que conlleva la proliferación del mundo digital, de un modelo dualista propio del siglo XX a uno pluralista o triangular del siglo XXI. En el modelo dualista, como su nombre lo indica, participan dos actores: los Estados territoriales y los oradores (individuos, medios masivos de comunicación y demás); en el modelo pluralista o triangular, se suman las empresas dueñas de la infraestructura del Internet, de manera destacada las de redes sociales que juegan un papel medular en la regulación de la libertad de expresión de sus usuarios.

El modelo dualista de regulación de la libertad de expresión se encuentra ampliamente desarrollado tanto en el ámbito internacional y nacional, con reglas claras sobre qué está permitido y qué no para los Estados e individuos.

En el plano internacional, el primer fundamento proviene de la Declaración Universal de los Derechos Humanos de las Naciones Unidas de 1948, cuyo preámbulo obliga a los Estados a

... "asegurar... el respeto universal y efectivo a los derechos y libertades fundamentales del hombre"; de manera que el artículo 19 de la misma De- 
Esta revista forma parte del acervo de la Biblioteca Jurídica Virtual del Instituto de Investigaciones Jurídicas de la UNAM

claración reconoce que "todo individuo tiene derecho a la libertad de opinión y de expresión; este derecho incluye el de no ser molestado a causa de sus opiniones, el de investigar y recibir informaciones y opiniones, y el de difundirlas, sin limitación" ...

La Declaración Universal obliga a los Estados a asegurar el respeto a los derechos humanos, incluida la libertad de expresión, que es concebida como el derecho a emitir opiniones, investigar, recibir información y difundirlas sin ser molestado y $\sin$ limitaciones.

El Pacto Internacional de Derechos Civiles y Políticos de 1966 desarrolla, a su vez, en su artículo 2o. las obligaciones de los Estados con relación a los derechos humanos en general, estableciendo tres obligaciones/ derechos para los Estados e individuos en materia de derechos civiles y políticos, a saber: respetar y garantizar los derechos previstos en el Pacto; adoptar las disposiciones legislativas o de otro tipo que hagan efectivos los derechos reconocidos en el Pacto; y garantizar la existencia de un recurso efectivo contra las violaciones a los derechos, que deberá ser resuelto por autoridad competente y cuya resolución será cumplida.

El artículo 19 del mismo Pacto señala los alcances del derecho a la libertad de expresión, estableciendo que abarca la libertad de buscar, recibir y difundir ideas y opiniones por cualquier medio posible. Además, se prevén una serie de restricciones a tal derecho, las que deben establecerse en la ley, es decir, hay una reserva de ley.

En el plano regional, se cuenta con dos instrumentos: la Declaración Americana de los Derechos y Deberes del Hombre de 1948 y la Convención Americana sobre Derechos Humanos de 1969. A lo que se suman las interpretaciones que ha realizado la Corte Interamericana de Derechos Humanos.

La Declaración Americana de los Derechos y Deberes del Hombre de 1948 señala en su artículo 4o. que "toda persona tiene derecho a la libertad... de opinión y de expresión y difusión del pensamiento por cualquier medio", esto es, se reconoce el derecho a la libertad de expresión y su difusión por cualquier medio.

La Convención Americana sobre Derechos Humanos de 1969 o Pacto de San José, por su parte, obliga en su artículo 1o. a los Estados a respetar los derechos y libertades, al tiempo de garantizar su ejercicio. El artículo 2o. compromete a los Estados a adoptar medidas legislativas para hacer efectivos los derechos y libertades consagrados en el mismo Pacto, entre 
Esta revista forma parte del acervo de la Biblioteca Jurídica Virtual del Instituto de Investigaciones Jurídicas de la UNAM

los que se encuentra la libertad de expresión. De manera que el artículo 13 del Pacto de San José se refiere al derecho a la libertad de expresión adicionando una serie de prohibiciones al marco jurídico internacional en la materia, como son la prohibición de: censura previa, de restringir la libertad de expresión por medios y vías indirectas, y de cierto tipo de propaganda.

Adicionalmente, la Corte Interamericana de Derechos Humanos ha efectuado una intensa labor interpretativa sobre los alcances del derecho a la libertad de expresión en el marco del artículo 13 del Pacto de San José. Por ejemplo, mediante la opinión consultiva OC-5/85 se determinó que son dos los pilares básicos para la interpretación del derecho a la libertad de expresión, el estándar democrático y el de dos dimensiones. Por el primero, el democrático, se hace hincapié en la importancia fundamental de la libertad de expresión para la existencia y sostenimiento del régimen democrático. Por el segundo, las dos dimensiones, se señala que el derecho a la libertad de expresión tiene implicaciones individuales y sociales, no sólo se protege la libertad de las personas sino también el derecho de la sociedad a conocer las distintas expresiones (Beroni, 2019).

En la sentencia Olmedo Bustos y otros vs Chile, la Corte Interamericana resolvió que la prohibición de censura previa contenida en el artículo 13.4 del Pacto es prácticamente absoluta (Beroni, 2019). Mientras que, en los casos Ivcher Bronstein vs Perú, Ríos y otros vs Venezuela, y Perozo y otros vs Venezuela, la Corte Interamericana se ha referido a los medios indirectos de violación de la libertad de expresión, señalando que éstos incluían, entre otros, a las nuevas tecnologías y los actos de particulares (Beroni, 2019).

Indudablemente el modelo de regulación dualista del siglo XX implica una serie de cuestiones como el reconocimiento mismo del derecho de libertad de expresión, sus alcances, limitaciones y, en contrapartida, la obligación de los Estados de garantizar y respetar el ejercicio de dicho derecho. Se trata a todas luces de un modelo que pone el acento en el papel del Estado como garante del derecho a la libertad de expresión de los sujetos. Sin embargo, los supuestos en que se basa este modelo regulatorio son cuestionados fuertemente ante el surgimiento del Internet y la infraestructura privada que lo controla.

En efecto, el siglo XXI se caracteriza porque son unos privados los que controlan la infraestructura del Internet y a través de esto la libertad de ex- 
Esta revista forma parte del acervo de la Biblioteca Jurídica Virtual del Instituto de Investigaciones Jurídicas de la UNAM

presión de los usuarios de la nueva plaza pública. Estas empresas de redes sociales son las que determinan qué se dice en el Internet a través de todo un aparato decisorio que incluye reglas, personas y desarrollos tecnológicos. El Estado es un simple espectador, con el agravante de que se ignoran por completo las reglas pensadas en el siglo XX para proteger la libertad de expresión a pesar de las declaraciones en contrario. Ante la incapacidad del Estado y sus actores para controlar la comunicación en línea y a las empresas de redes sociales, a diario éstas eliminan miles de cuentas y millones de publicaciones, todo esto sin garantía de audiencia, en la mayor opacidad e incluso como una forma de censura previa.

El modelo de regulación del siglo XXI es entonces un triángulo, como señala Jack M. Balkin (2018a). La libertad de expresión de los sujetos ahora es regulada de manera paralela tanto por el Estado y el modelo del siglo XX, como por las empresas de redes sociales y sus modelos de autorregulación. Lo que significa, sin lugar a dudas, un cambio de enormes dimensiones respecto a la forma en que se concibe, conceptualiza, discute y regula la libertad de expresión.

En la actualidad la libertad de expresión fuera del mundo en línea transcurre en gran medida conforme al modelo regulatorio del siglo XX, en el que existe todo un entramado jurídico que garantiza el derecho a la libertad de expresión y que obliga al Estado a hacerlo efectivo y respetarlo. Al mismo tiempo, la libertad de expresión en el Internet está sometida por completo a la regulación de las empresas de redes sociales, sin mayor límite que el que ellas mismas se imponen y con un poder sin parangón sobre lo que dicen los sujetos.

Hay una coexistencia de dos tipos de reguladores, los Estados y las empresas de redes sociales, con incidencia en los distintos ámbitos de la vida de los sujetos, fuera de línea y en Internet. Más importante, en el primer caso, la regulación de los Estados y fuera de línea, entraña una serie de reglas jurídicas ampliamente desarrolladas sobre el derecho a la libertad de expresión, sus alcances y limitaciones. En el segundo caso, la regulación de las empresas en Internet, se está en presencia de una serie de reglas privadas, que casi nadie conoce y, más trascendente, que norman los alcances y límites a la libertad de expresión sin restricción alguna para las empresas y colocando en la más completa indefensión a quienes usan las redes sociales. 
Esta revista forma parte del acervo de la Biblioteca Jurídica Virtual del Instituto de Investigaciones Jurídicas de la UNAM

En un entorno en el que más seres humanos tienen acceso al Internet y en el que cada vez dedican mayor tiempo a su uso, adquiere gran relevancia la reflexión en torno a la libertad de expresión en línea y la necesidad de acotar en esta materia a las empresas de redes sociales El debate es apenas incipiente, aunque surgen atisbos de que la respuesta a este desafío puede provenir del derecho internacional de los derechos humanos como se expondrá enseguida.

\section{LA LIBERTAD DE EXPRESIÓN EN INTERNET Y EL DERECHO INTERNACIONAL DE LOS DERECHOS HUMANOS}

El desafío que para el derecho significan el Internet y las redes sociales en términos de la necesidad de adecuar el marco jurídico imperante no es, en modo alguno, novedoso. Antes que ellos, otros cambios tecnológicos también representaron diversos retos que requirieron de ajustes legales; el telégrafo, las cámaras portátiles y las computadoras son sólo algunos ejemplos de tecnologías de las comunicaciones que dieron lugar a modificaciones en el entramado jurídico nacional e internacional (Gasser, 2016).

Lo que sí es peculiar al Internet y las redes sociales es el tipo de respuesta que habrá de darse desde el derecho. A este respecto, Urs Gasser (2016: 64) identifica, a grandes rasgos, tres patrones de respuesta de la ley ante los desafíos tecnológicos:

(1) Cuando se trata de tecnologías innovadoras, el sistema legal... por defecto busca aplicar las viejas reglas al problema resultante de la nueva tecnología y sus usos (subsunción)...

(2) Cuando la subsunción se considera insuficiente dada la novedad de los problemas planteados por la nueva tecnología, el sistema legal podría recurrir a la innovación dentro de su propio sistema... (innovación gradual) ...

(3) Un más radical enfoque de cambio de paradigma es una reforma de la ley más profunda en la que no sólo se actualizan las normas individuales, sino que también se cambian enfoques o instrumentos completos...

En el caso de las redes sociales y la libertad de expresión el debate se ha centrado, hasta ahora, en las posibilidades de aplicar la normatividad internacional de derechos humanos sobre libertad de expresión a la regulación 
Esta revista forma parte del acervo de la Biblioteca Jurídica Virtual del Instituto de Investigaciones Jurídicas de la UNAM

empresarial de contenidos en línea. La Comisión Interamericana de Derechos Humanos (2017: 38) se manifestó en dicho sentido al señalar que:

82. La Comisión Interamericana ha sostenido desde hace más de una década que "el derecho a la libertad de expresión en los términos consagrados por el artículo 13 de la Convención Americana protege de igual manera tanto a los medios de comunicación tradicionales como a la expresión difundida a través de Internet".

83. La Declaración Conjunta sobre Libertad de Expresión e Internet prevé como principio general que "la libertad de expresión se aplica a Internet del mismo modo que a todos los medios de comunicación. Las restricciones a la libertad de expresión en Internet solo resultan aceptables cuando cumplen con los estándares internacionales que disponen, entre otras cosas, que deberán estar previstas por la ley y perseguir una finalidad legítima reconocida por el derecho internacional y ser necesarias para alcanzar dicha finalidad (la prueba "tripartita").

El Informe del Relator Especial sobre la promoción y protección del derecho a la libertad de opinión y de expresión (2018: 16 y 17) abordó expresamente la regulación de los contenidos en línea por las empresas de redes sociales, recomendando también la aplicación del marco jurídico internacional de los derechos humanos en los términos siguientes:

Principios de derechos humanos para la moderación del contenido por parte de las empresas

41. Las normas privadas, que varían en función del modelo de negocio de cada empresa y las vagas afirmaciones acerca de los intereses de la comunidad, han creado un entorno inestable, imprevisible e inseguro para los usuarios... Las leyes nacionales no son adecuadas para las empresas que buscan normas comunes para una base de usuarios que es geográfica y culturalmente diversa. Sin embargo, las normas de derechos humanos, si se aplican de manera transparente y coherente, con aportaciones pertinentes de la sociedad civil y los usuarios, proporcionan un marco para la responsabilidad de los Estados y las empresas ante los usuarios a través de las fronteras nacionales.

42. Un marco de derechos humanos facilita la aplicación de respuestas normativas frente a las restricciones estatales excesivas - siempre que las empresas se rijan por normas similares-...

43. Los principios de derechos humanos también permiten a las empresas crear un entorno incluyente que dé cabida a las diversas necesidades 
Esta revista forma parte del acervo de la Biblioteca Jurídica Virtual del Instituto de Investigaciones Jurídicas de la UNAM

e intereses de sus usuarios, estableciendo al mismo tiempo unas normas básicas de comportamiento previsibles y coherentes. En medio de un creciente debate sobre si las empresas ejercen una combinación de funciones de intermediación y edición, el derecho de los derechos humanos ofrece a los usuarios la promesa de que pueden contar con unas normas fundamentales para proteger su libertad de expresión más allá de lo que la legislación nacional podría limitar...

La Segunda Sala de la Suprema Corte de Justicia de México se ha pronunciado en un sentido similar como obra en la tesis aislada LIBERTAD DE EXPRESIÓN Y OPINIÓN EJERCIDAS A TRAVÉS DE LA RED ELECTRÓNICA(INTERNET). RESTRICCIONES PERMISIBLES, donde se señala lo que sigue:

Las restricciones a determinados tipos de información o expresión admitidas en virtud del derecho internacional de los derechos humanos, también resultan aplicables a los contenidos de los sitios de Internet. En consecuencia, para que las limitaciones al derecho humano referido ejercido a través de una página web, puedan considerarse apegadas al parámetro de regularidad constitucional, resulta indispensable que deban: (I) estar previstas por la ley; (II) basarse en un fin legítimo; y (III) ser necesarias y proporcionales. ${ }^{6}$

El relator especial sobre la libertad de opinión y expresión de la Organización de las Naciones Unidas (ONU), David Kaye (2019), ha ahondado más en el tema proponiendo que las empresas de redes sociales adopten explícitamente la normatividad internacional de derechos humanos en sus reglas sobre moderación de contenidos, para cuya interpretación se podrían auxiliar de la basta jurisprudencia sobre la materia derivada de las decisiones de las cortes internacionales. A lo que se suman planteamientos relacionados con la mejora radical de la transparencia sobre sus acciones en el ámbito de la regulación de contenidos, y una mayor rendición de cuentas con base en la creciente colaboración y participación de líderes de la sociedad civil, activistas y académicos. Los gobiernos, según esta visión, deben regular aspectos tales como el monitoreo del comportamiento de las empresas de redes sociales en materia de libertad de expresión de sus usuarios, la protección del espacio para la expresión individual, reforzar las acciones de transparencia de las compañías, entre otros.

6 Tesis 2a. CV/2017 (10a), Gaceta del Semanario Judicial de la Federación, décima Época, Libro 43, t. II, junio de 2017, p. 1439, registro digital: 2012519. 
Esta revista forma parte del acervo de la Biblioteca Jurídica Virtual del Instituto de Investigaciones Jurídicas de la UNAM

No obstante, existen opiniones que ponen en entredicho la viabilidad de aplicar el marco internacional de los derechos humanos a la regulación de contenidos por las redes sociales. Los señalamientos abarcan una serie de aspectos como (Douek, 2018): las contradicciones que pudiesen presentarse entre las leyes de los distintos países y el marco jurídico internacional; el carácter privado de las empresas de redes sociales $\mathrm{y}$, por tanto, la no aplicabilidad de las disposiciones del derecho internacional de los derechos humanos; la naturaleza no vinculante de los principios rectores de la ONU sobre empresas y derechos humanos; la diversidad de la normatividad internacional y las interpretaciones incluso contradictorias de su contenido; los problemas derivados de no tomar en cuenta los contextos locales; y, en general, el choque entre la prohibición absoluta de intervención estatal que prevé la primera enmienda estadounidense y el enfoque más intrusivo de la normatividad internacional en materia de libertad de expresión.

La discusión sobre la aplicación de la normatividad internacional de derechos humanos a la regulación empresarial de contenidos en línea está en ciernes. Si bien hay avances como la posibilidad de que el Consejo Asesor de Contenido de Facebook tome en cuenta dicha normatividad cuando revise la eliminación de contenido de la plataforma (artículo 2o. del Acta Constitutiva del Consejo Asesor), ${ }^{7}$ faltan por definirse las acciones que tomarán los otros gigantes de las redes sociales y, más importante, el papel que desempeñarán los Estados. El debate sigue abierto y este artículo constituye una primera aproximación a la reflexión en torno a la libertad de expresión en los tiempos del Internet y las redes sociales.

\section{CONCLUSIONES}

El incremento vertiginoso en las últimas casi tres décadas en el número de usuarios y el tiempo que éstos dedican a navegar por el Internet o más precisamente en las redes sociales, ha trastocado fuertemente la manera en que las personas se comunican e interactúan. El Internet y las redes sociales se han convertido en la nueva plaza pública, el espacio donde las personas comparten sus opiniones, mensajes, videos, fotos y demás.

7 Disponible en: https://www.oversightboard.com/governance/.

Cuestiones Constitucionales, Núm. 44, Enero-Junio 2021

ISSN: $2448-4881$ 
Esta revista forma parte del acervo de la Biblioteca Jurídica Virtual del Instituto de Investigaciones Jurídicas de la UNAM

Esta creciente importancia del Internet en la vida diaria de las personas ha ido acompañada por una concentración de su infraestructura en unas cuantas empresas de redes sociales. Atrás quedó el ideal de un Internet libre y descentralizado, en su lugar un puñado de empresas controlan la infraestructura en la que navegan millones de personas alrededor del orbe. Concentración que ha dado lugar a que este pequeño número de empresas, principalmente de redes sociales, tengan un enorme poder para decidir qué se puede publicar, compartir, opinar en línea. Son los guardianes de la libertad de expresión en Internet.

De tal manera que, en su calidad de guardianes de la libertad de expresión en Internet, estas empresas de redes sociales han puesto en marcha todo un sistema privado de revisión de contenidos, que incluyen una serie de reglas, procedimientos, personas y tecnologías de punta, por medio de los cuales deciden qué se dice en línea. Se trata de un sistema regulatorio privado de la libertad de expresión en Internet, con alcance para los millones de usuarios de las redes sociales, que es opaco, no le rinde cuentas a nadie, no respeta las garantías mínimas procesales y que, por tanto, deja en la indefensión a los sujetos.

La regulación estatal de la libertad de expresión propia del siglo XX coexiste ahora con un sistema privado de regulación aplicable al Internet. El modelo regulatorio de la libertad de expresión es un triángulo, donde los individuos están sometidos al mismo tiempo a la regulación estatal aplicable fuera de línea y a la regulación de las redes sociales para el Internet.

Es un cambio de enorme profundidad en materia de la libertad de expresión que llama a emprender la reflexión respecto sus alcances, implicaciones y ajustes jurídicos necesarios. La respuesta no se ha hecho esperar ante el creciente y discrecional poder que detentan las empresas de redes sociales en el ámbito de la libertad de expresión de sus millones de usuarios, por lo que se ha planteado limitar tal poder mediante la aplicación del derecho internacional de los derechos humanos.

El artículo es un primer intento por poner sobre la mesa la reflexión sobre este tema, quedan pendientes todo un conjunto de aspectos tales como cuál es la respuesta más adecuada frente a este desafío tecnológico, el papel de los Estados, del derecho y de las mismas empresas de redes sociales. 
Esta revista forma parte del acervo de la Biblioteca Jurídica Virtual del Instituto de Investigaciones Jurídicas de la UNAM

\section{FUENTES CONSULTADAS}

BALKIN, Jack M. 2018a. Free speech is a triangle [versión electrónica]. Columbia Law Review. Vol. 118 (7).

BALKIN, Jack M. 2018b. Free speech in the algorithmic society: big data, private governance, and the new school of regulation [versión electrónica]. UC Davis Law Review. Vol. 51 (3).

BERONI, Eduardo et al. 2019. Libertad de pensamiento y expresión. En STEINER, Christian y FUCHS, Marie-Christine (eds.). Convención americana sobre derechos humanos. Comentario. Bogotá, Colombia: Konrad Adenauer Stiftung.

Calcaneo Monts, Mauricio Augusto. 2019. Big data, big data analytics y datos personales en los tiempos del Internet: de la autorregulación estadounidense al reglamento general de protección de datos de la Unión Europea [versión electrónica]. Estudios en Derecho a la Información. Núm. 8.

Citrón, Danielle Keats y RichARdS, Neil M. 2018. Four principles for digital expression (You won't believe \#3!) [version electronica]. Washington University Law Review. Vol. 95.

CLEGG, Nick. 2019. Draft charter: An oversight board for content decisions. Recuperado el 17 de agosto de 2019. Disponible en: https:// fbnewsroomus. files.wordpress.com/2019/01/draft-charter-oversightboard-for-content-decisions-2.pdf.

Comisión Interamericana de Derechos Humanos-Relatoría Especial para la Libertad de Expresión. 2017. Estándares para una Internet libre, abierta e incluyente. Recuperado el 2 de septiembre de 2019, disponible en: http://www.oas.org/es/cidh/expresion/docs/publicaciones/internet_2016_esp.pdf.

CONGER, Kate, y DAVEY, Alba. 2020. Twitter Refutes Inaccuracies in Trump's Tweets About Mail-In Voting. The New York Times. Recuperado el 5 de septiembre de 2020, disponible en: https://www.nytimes. com/2020/05/26/technology/twitter-trump-mail-in-ballots.html.

DiAMOND, Larry. 2010. Liberation technology [version electronica]. Journal of Democracy. Vol. 21 (3).

DiAMOND, Larry. 2019. The threat of postmodern totalitarism [versión electrónica]. Journal of Democracy. Vol. 30 (1).

Cuestiones Constitucionales, Núm. 44, Enero-Junio 2021

ISSN: $2448-4881$ 
Esta revista forma parte del acervo de la Biblioteca Jurídica Virtual del Instituto de Investigaciones Jurídicas de la UNAM

DoDDS, Laurence. 2020. Twitter bans Donald Trump's campaign from tweeting until he deletes false coronavirus claim. The Telegraph. Recuperado el 5 de septiembre de 2020, disponible en: https://www.tel egraph.co.uk/technology/2020/08/06/twitter-gags-donald-trumps-cam paign-account-deletes-false-covid/.

DoueK, Evelyn. 2018. U.N. Special Rapporteur's Latest Report on Online Content Regulation Calls for "Human Rights by Default". Lawfare blog. Recuperado el 3 de septiembre de 2020, disponible en: https:// www.lawfareblog.com/un-special-rapporteurs-latest-report-onlinecontent-regulation-calls-human-rights-default.

Facebook. 2019. Global feedback and input on the Facebook oversight board for content decisions. Recuperado el 20 de agosto de 2019, disponible en: https://fbnewsroomus.files.wordpress.com/2019/06/over sight-board-consultation-report-2.pdf.

Freedom House. 2018. Freedom on the net 2018. The rise of digital authoritarianism. Washington D.C.: Freedom House. Recuperado el 30 de julio de 2019, disponible en: https://freedomhouse.org/sites/default/ files/2020-02/10192018_FOTN_2018_Final_Booklet.pdf.

GASSER, Urs. 2016. Recoding privacy law: reflections on the future relationship among law, technology, and privacy [versión electrónica]. Harvard Law Review. Vol. 130 (2).

KAYE, David. 2019. A new constitution for content moderation. Medium One Zero. Recuperado el 5 de septiembre de 2020, disponible en: $h t t$ ps://onezero.medium.com/a-new-constitution-for-content-moderation$6249 a f 611 b d f$.

KLONICK, Kate. 2018. The new governors: the people, rules and processes governing online speech [versión electrónica]. Harvard Law Review. Vol. 131 (6), 1598-1670.

Relator Especial sobre la promoción y protección del derecho a la libertad de opinión y de expresión. 2018. Informe del Relator Especial sobre la promoción y protección del derecho a la libertad de opinión y de expresión. Recuperado el 5 de septiembre de 2020, disponible en: https:// www.undocs.org/es/A/HRC/38/35.

ZITTRAIN, Jonathan. 2018. Fixing the Internet [versión electrónica]. Science. Vol. 362 (6417). 
Esta revista forma parte del acervo de la Biblioteca Jurídica Virtual del Instituto de Investigaciones Jurídicas de la UNAM

ZuCKERBERG, Mark. 2019. The Internet needs new rules. Let's start in these four areas. The Washington Post. Recuperado el 12 de agosto de 2019, disponible en: https://www.washingtonpost.com/opinions/ mark-zuckerberg-the-internet-needs-new-rules-lets-start-in-these-fourareas/2019/03/29/9e6f0504-521a-11e9-a3f7-78b7525a8d5f_story. html?utm_term $=.5805760 f 9827$.

ZuCKERBERG, Mark. 2018. A blueprint for content governance and enforcement [en línea]. Recuperado el 13 de agosto de 2019, disponible en: https://www.facebook.com/notes/mark-zuckerberg/a-blueprintfor-content-governance-and-enforcement/10156443129621634/?hc location $=u f$. 